\title{
Indicadores ecológicos de hábitat y biodiversidad en un paisaje neotropical: perspectiva multitaxonómica
}

\author{
Noel González-Valdivia ${ }^{1}$, Susana Ochoa-Gaona ${ }^{1 *}$, Carmen Pozo $^{2}$, Bruce Gordon Ferguson $^{3}$, \\ Luis José Rangel-Ruiz ${ }^{4}$, Stefan Louis Arriaga-Weiss ${ }^{4}$, Alejandro Ponce-Mendoza ${ }^{1}$ \\ \& Christian Kampichler ${ }^{4}$ \\ 1. El Colegio de la Frontera Sur, Sistemas de Producción Alternativos. Apdo. Postal 1042, Admón. de Correos de \\ Tabasco 2000,86031 Villahermosa, Tabasco, México; siankaan2003@gmail.com, sochoa@ecosur.mx \\ 2. El Colegio de la Frontera Sur. Conservación de la Biodiversidad.Chetumal, Quintana Roo, México; cpozo@ecosur.mx \\ 3. El Colegio de la Frontera Sur, Sistemas de Producción Alternativos. San Cristóbal de las Casas, Chiapas, México; \\ bferguson@ecosur.mx \\ 4. División de Ciencias Biológicas. Universidad Juárez Autónoma de Tabasco. Villahermosa, Tabasco, México; \\ christian.kampichler@web.de, slaw2000@prodigy.net.mx, ljrangel@cicea.ujat.mx \\ * Correspondencia
}

Recibido 03-IX-2010. Corregido 16-I-2011. Aceptado 17-II-2011.

\begin{abstract}
Ecological indicators of habitat and biodiversity in a Neotropical landscape: multitaxonomic perspective. The use of indicator species to characterize specific ecological areas is of high importance in conservation/restoration biology. The objective of this study was to identify indicator species of diverse taxa that characterize different landscape units, and to better understand how management alters species composition. We identified two ecomosaics, tropical rain forest and the agricultural matrix, each one comprised of four landscape units. The taxonomic groups studied included birds (highly mobile), butterflies (moderately mobile), terrestrial gastropods (less mobile) and trees (sessile). Sampling efficiency for both ecomosaics was $\geq 86 \%$. We found 50 mollusks, 74 butterflies, 218 birds and 172 tree species, for a total of 514 species. Using ordination and cluster analysis, we distinguished three habitat types in the landscape: tropical rainforest, secondary vegetation and pastures with scattered trees and live fences. The InVal $(\geq 50 \%)$ method identified 107 indicator species, including 45 tree species, 38 birds, 14 butterflies and 10 gastropods. Of these, 35 trees, 10 birds, four butterflies and eight gastropods were forest indicators. Additionally, 10, 28, 10 and two species, respectively per group, were characteristic of the agricultural matrix. Our results revealed a pattern of diversity decrease of indicator species along the rainforest-secondary forest-pasture gradient. In the forest, the gastropods Carychium exiguum, Coelocentrum turris, Glyphyalinia aff. indentata y Helicina oweniana were significantly correlated $(\mathrm{p}<0.05)$ with $90 \%$ of the other groups of flora and fauna indicator species. These findings suggest that gastropods may be good indicators of forest habitat quality and biodiversity. The secondary vegetation is an intermediate disturbance phase that fosters high diversity in the agricultural matrix. We exemplify a multitaxa approach, including mesofauna, for ecological monitoring of agricultural landscapes. Rev. Biol. Trop. 59 (3): 1433-1451. Epub 2011 September 01.
\end{abstract}

Key words: biodiversity, cultural landscapes, habitat groups, indicator species, rainforest, Mexico.

La conservación de especies silvestres enfrenta el reto de su mantenimiento frente a una realidad caracterizada por la presión antropogénica sobre los ecosistemas terrestres (Saunders et al. 1991, Bustamante \& Grez 1995), lo que amenaza con degradar, disminuir e incluso desaparecer los remanentes de hábitats nativos aún disponibles (Murcia 1995). Por esto, los sistemas naturales y su biota deben ser primeramente identificados y cuantificados para su comprensión y preservación (Villavicencio-Enríquez \& Valdez-Hernández 2003), con el problema adicional de la carencia de recursos suficientes para catalogar toda la 
biodiversidad a proteger en estos ecosistemas (Beever et al. 2006). En este sentido la comunidad internacional se ha organizado para discutir estrategias para la conservación de la biodiversidad (COP-10 Biodiversidad 2010) y ha establecido criterios para determinar el nivel de riesgo de la misma (CITES 1973).

De manera complementaria, se ha considerado que la conservación biológica debe contar con una cantidad apropiada de áreas eficientemente protegidas (Turner 1996) alternadas con matrices de uso de suelo de alta calidad ecológica, que faciliten el refugio de la flora y fauna silvestre (Perfecto \& Vandermeer 2002, Cook et al. 2004, Kupfer et al. 2006). En áreas tropicales, estos enfoques incluyen como estrategia de manejo adaptativo a la agroforestería en el diseño de paisajes (Forman \& Godron 1986, Turner et al. 2001, Villavicencio-Enríquez \& Valdez-Hernández 2003, Beever et al. 2006) y la restauración estructural y funcional de los ecosistemas que permitan su resiliencia, es decir recuperarse o reorganizarse después de periodos de perturbación causados por el ser humano y los agentes naturales (Walker et al. 2002, Higgs 2003, Chazdon 2008).

Aunque recientemente se ha dado mayor atención al estudio de la persistencia de la flora y fauna silvestre en paisajes culturales (Driscoll 2005, Castellón \& Sieving 2006, Chazdon et al. 2008, Perfecto \& Vandermeer 2008), el tema en el trópico aún es insuficiente (Schulze et al. 2004). Esto limita la comprensión de las relaciones socio-ecológicas que se desarrollan en el Neotrópico, e impide contar con insumos para la adecuada formulación de políticas de desarrollo, que concilien los objetivos de producción con los de conservación (Holling 2001, Toledo \& Barrera-Bassols 2008).

Para investigar la permanencia de las comunidades de organismos terrestres nativos en paisajes culturales, se han utilizado indicadores ecológicos seleccionados debido a que son bien conocidos taxonómicamente, son fáciles de muestrear e identificar, se presentan en diferentes condiciones ambientales y muestran fuertes relaciones con otros grupos biológicos de interés (Noss 1990, Spellerberg 2005, Heino et al. 2009), con lo que se disminuyen los costos que representa hacer inventarios totales. La estrategia ideal involucra la combinación de distintos grupos biológicos o comunidades (Metzger 2006) que presenten varios niveles de sensibilidad para medir el impacto en la biota por la acción humana a escala de paisaje.

La evaluación del papel de los paisajes culturales en la conservación de la biodiversidad solo resulta útil si se compara con ecosistemas conservados que sirven como referentes ecológicos para conocer cómo se estructuran las especies nativas (sensu Higgs 2003). Este método, combina abordajes provenientes de multidisciplinas sintéticas como la biología de la conservación, la ecología del paisaje y la restauración ecológica, que pretenden aportar resultados que cumplan con una visión holística (Dobson et al. 1997, Young 2000, Jobin et al. 2003, Holl \& Crone 2004).

El objetivo de este estudio fue el de identificar, desde una perspectiva multitaxonómica, las especies indicadoras que caracterizan las diferentes unidades del paisaje, y conocer cómo el manejo humano altera la composición de especies. Por lo tanto, se espera que las especies identificadas se conviertan en una herramienta para el monitoreo de la calidad del hábitat del paisaje del Área Natural Protegida Cañón del Usumacinta y de las selvas adyacentes de El Petén y La Selva Lacandona.

\section{MATERIALES Y MÉTODOS}

Zona de estudio: El estudio se llevó a cabo en el ejido Niños Héroes de Chapultepec (en adelante "ejido"), municipio de Tenosique, Estado de Tabasco, Sureste de México, que se localiza entre las coordenadas $17^{\circ} 15^{\prime} 00^{\prime \prime}$

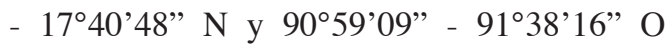
(Ochoa-Gaona et al. 2008). El clima es cálido y húmedo, con $26^{\circ} \mathrm{C}$ como promedio anual de temperatura y $2750 \mathrm{~mm}$ como precipitación media anual (Isaac-Márquez et al. 2005). El relieve de valles entre montañas tiene una altura que fluctúa entre 200 y $700 \mathrm{msnm}$. El paisaje se extiende sobre una superficie de 2 066ha. 
La vegetación de referencia es de tipo bosque tropical lluvioso según la propuesta de Ellenberg \& Mueller-Dombois (1967), dominado por especies de las familias Sapotaceae, Moraceae, Fabaceae y Apocynaceae en el dosel (Rzedowski 2006). El sotobosque arbustivo está dominado por especies de: Rubiaceae, Violaceae y Arecaceae, mientras el herbáceo por: Araceae, Zingiberaceae y Pteridophyta. El bosque fue sometido a extracción de cedro (Cedrela odorata) y caoba (Swietenia macrophylla) desde la década de 1970 (Gobierno del Estado 1997).

En el paisaje se diferencia una matriz agropecuaria generada por la actividad agrícola, que inició con la roza-tumba-quema, y que ha sido desplazada gradualmente hacia la ganadería extensiva dejando un remanente de bosque como reserva forestal (Isaac-Márquez et al. 2005). Como resultado, la matriz agropecuaria es un ecomosaico con un patrón de pasturas con árboles dispersos y en línea, con vegetación secundaria joven y vegetación secundaria madura. En la parte alta de las montañas y dispersas entre la matriz se presenta un ecomosaico de unidades de bosque que constituye el referente ecológico. El ejido forma parte del Área Protegida Cañón del Usumacinta (Diario Oficial de la Federación 2008).

Unidades de paisaje: Se diferenciaron dos ecomosaicos, el bosque tropical lluvioso (en adelante RE, por ser referente ecológico) y la matriz agropecuaria (en adelante MA). Dentro del ecomosaico de la matriz agropecuaria se distinguieron cuatro unidades de paisaje (sensu Zonneveld 1989) que incluyen a la vegetación secundaria (también conocidos como barbechos, tacotales, guamiles o acahuales) madura de $\geq 15$ años de edad (AM), ubicados generalmente en la parte superior de las lomas, las unidades de vegetación secundaria o acahuales jóvenes de $<15$ años de edad (AJ) comúnmente ubicados sobre las laderas y hacia el pie de las lomas y adyacentes a unidades de potreros con árboles dispersos (PAD) y árboles en línea (AL), que ocupan las partes planas o ligeramente onduladas del ejido.
En el bosque se seleccionaron cuatro unidades, de manera que correspondieran con la distribución de las unidades de la matriz agropecuaria para tenerlas como sus referentes ecológicos. De esta manera se tuvieron unidades de bosque establecido en la parte superior de las montañas como referente de la vegetación secundaria o acahuales maduros (REAM), seguidas por las de bosque sobre laderas como referente de la vegetación secundaria o acahuales jóvenes (REAJ), el bosque al pie de los montes y partes planas como referente de los potreros (REPAD), y como referente de los árboles en línea se muestrearon los caminos de acceso al interior del bosque (REL). Las unidades de paisaje de cada ecomosaico se replicaron cuatro veces.

Grupos de indicadores: Se seleccionaron tres grupos de fauna reconocidos por su valor como indicadores ecológicos. Estos representaron tres niveles de movilidad o vagilidad considerados como representativos de la gama de sensibilidades que la fauna tiene ante cambios en las condiciones del hábitat (Wilcox et al. 1986): muy móviles (aves), moderadamente móviles (mariposas Nymphalidae), y poco móviles (gasterópodos terrestres). Los árboles fueron el componente sésil del estudio. Se espera que los organismos con menor capacidad de movimiento, sean más sensibles a la perturbación. Los grupos biológicos escogidos han sido destacados por numerosos estudios como indicadores de tipo ecológico, útiles para monitorear la calidad de los hábitats y los cambios de su calidad por efecto de la perturbación antropogénica. Al respecto, las aves han sido mencionadas por Kattan et al. (1994) y Stouffer \& Bierregaard (1995), las Nymphalidae frugívoras por Tobar et al. (2007), Marín et al. (2009) y Pozo et al. (2009), y los moluscos terrestres por Berger \& Dallinger (1993), Secrest et al. (1996), Vera-Ardila \& Linares (2005), Baqueiro-Cárdenas et al. (2007) y Pérez et al. (2007). La flora arbórea (DAP $\geq 5 \mathrm{~cm}$ ) constituyó una cuarta comunidad indicadora que además influye en las características estructurales y ambientales de las unidades de paisaje (Ramírez-Marcial et al. 2001, Ochoa-Gaona et al. 2004). 
Muestreo: Cada grupo de fauna fue muestreado durante el año 2008. Para árboles se modificó la propuesta de Ochoa-Gaona et al. (2004) y se establecieron 96 parcelas rectangulares de $5 \times 100 \mathrm{~m}$ en las que se registraron todos los individuos $(\mathrm{DAP} \geq 5 \mathrm{~cm})$ que tuviesen al menos la mitad de su base dentro del perímetro de la parcela, con este esquema se evaluaron 6 $000 \mathrm{~m}^{2}$ por unidad del paisaje. La ornitofauna se evaluó mediante rutas con 12 puntos de conteo con 25m de radio fijo (Bibby et al. 1998), completando 48 puntos por unidad del paisaje en cada estación evaluada (seca: febrero a abril y lluviosa: julio a septiembre), cada punto se observó por $10 \mathrm{~min}$. La malacofauna terrestre se registró durante el periodo lluvioso, siguiendo la propuesta de Rangel-Ruiz \& GamboaAguilar (2000, 2006), de establecer por cada unidad un total de 40 parcelas de $1 \mathrm{~m}^{2}$ tomando muestras de suelo hasta $5 \mathrm{~cm}$ de profundidad y 40 estaciones de búsqueda por tiempo (15 min de revisión) en las que se revisaron en un radio de $10 \mathrm{~m}$ a partir de cada parcela, las plantas, troncos caídos o en pie, rocas y la superficie del suelo para recolectar macromoluscos (González-Valdivia et al. 2010). Las Nymphalidae frugívoras fueron capturadas por medio de trampas Van Someren-Rydon cada 50m sobre un transecto de $500 \mathrm{~m}$ de longitud y $25 \mathrm{~m}$ de ancho, totalizando 40 trampas en cada unidad de paisaje (Pozo et al. 2008); esto se realizó durante la época lluviosa, periodo de mayor fructificación de la flora arbórea local (OchoaGaona et al. 2008). En los lugares abiertos se utilizaron los árboles dispersos para colgar las trampas. El cebo, fermentado de piña, banano y cerveza, fue reemplazado diariamente para evitar sesgos debidos a agentes climáticos o por acción de animales, y mantener constante la calidad del mismo. Las mariposas se retiraron dos veces al día al reponer el cebo.

Sólo se usó el número de registro de individuos, debido a que cada grupo utiliza diferente técnica de muestreo y no es posible homologar las unidades para calcular abundancias por unidad de área para hacer comparaciones.

La identificación taxonómica de las especies se realizó con apoyo de especialistas del
Museo de Zoología de ECOSUR Quintana Roo (Nymphalidae) los especímenes se depositaron en la Colección de Lepidóptera registro INE QNR.IN.018.0497. Para gasterópodos se recibió apoyo de especialistas del Laboratorio de Malacología adscrito a la División de Ciencias Biológicas de la Universidad Juárez Autónoma de Tabasco (UJAT) donde se depositaron los especímenes. La determinación botánica se hizo por comparación con material de referencia de los Herbarios de ECOSUR en San Cristóbal de las Casas, Chiapas y de la UJAT en Villahermosa, Tabasco, los especímenes se depositaron en el herbario de la UJAT. Las aves fueron identificadas en campo mediante el cotejo con las guías ilustradas de Howell \& Webb (2005) y Van Perlo (2006).

Análisis estadístico: Con base en la estructura total de las especies de cada comunidad (riqueza se especies) y el registro de individuos de cada especie, se estimaron los índices ecológicos de diversidad Shannon-Wiener, dominancia de Simpson y equitatividad de Pielou siguiendo la sugerencia de Moreno (2001) y Pérez et al. (2007). Para éstos cálculos se utilizó EstimateS 8.0 (Colwell 2008). El porcentaje de detección (Efi) se calculó mediante el uso de porcentaje del total de especies observadas con relación al total de especies esperadas en cada unidad y ecomosaico. Asimismo, se promediaron los índices de diversidad, dominancia y equitatividad siguiendo el esquema de trabajo de Castellanos \& Serrato (2008).

Se hizo una exploración de los datos mediante análisis de ordenación dimensional no métrica (NMDS) para evaluar la agrupación de las unidades de paisaje del ejido en función del total de organismos considerados. Posteriormente se hizo análisis de cúmulos con el algoritmo de grupos apareados (UPGMA) utilizando como medida de distancia al índice Bray-Curtis, que minimiza el efecto de los doble ceros (Zuur et al. 2007). El agrupamiento y la ordenación utilizando PAST 2.0 que es de acceso libre y con la misma robustez que otros paquetes estadísticos (Hammer et al. 2001), permitieron determinar el patrón de 
diferenciación entre las unidades de paisaje, expresado a través de modificaciones en la composición estructural registrada en el interior de las mismas. Estos patrones de diferenciación o agrupamiento fueron la base para identificar condiciones generales de ambiente y estructura, que fueron denominados categorías de hábitat.

Una vez diferenciados los tipos de hábitats mediante la ordenación y la agrupación, se procedió a identificar las especies indicadoras de cada condición de hábitat mediante el método de asignación del Valor de Indicador o InVal propuesto por Dufrene \& Lengendre (1997). Este método consiste en una combinación de medidas de especificidad y fidelidad de hábitat (McGeoch et al. 2002) que permite distinguir aquellas especies que tienen la mayor afinidad por los diferentes hábitats presentes en un territorio bajo estudio. La herramienta InVal permite identificar a aquellos táxones asociados con mayor peso a las diferentes unidades que configuran el paisaje del ejido, siendo de mayor relevancia aquellas que identifican al bosque, como ecosistema de referencia para la zona. Las especies consideradas como características (indicadoras) de una condición de hábitat fueron aquellas con InVal $\geq 50 \%$, tal como sugieren Tejeda-Cruz et al. (2008) y que presentaron una alta significación estadística en la prueba de Monte Carlo, con 1000 iteraciones aleatorias. El programa utilizado en este procedimiento fue PcOrd 4.25 (McCune \& Mefford 1999).

Siguiendo a Schulze et al. (2004) y a Heino et al. (2009), para evaluar la posible existencia de relaciones entre las diferentes especies indicadoras del bosque, se aplicó una correlación de Pearson, mediante el uso del número de registros de los individuos con un InVal $\geq 50 \%$, se usó esta porque permite relacionar dos variables de manera adimensional (Ruiz-Díaz et al. 1998). De tal forma, que se seleccionó el bosque para este análisis, debido a que es el ecosistema a conservar. Con esto, se buscó determinar la existencia de especies que al correlacionarse con la mayoría, puedan funcionar como indicadoras de la biodiversidad. Aquellas especies - que previamente fueron identificadas como indicadoras del bosque
- cuyo número de registros de individuos se correlaciona significativamente con la mayoría de las especies indicadoras de los otros grupos biológicos, se consideraron como especies indicadoras de biodiversidad de dicho hábitat.

\section{RESULTADOS}

El porcentaje de detección (Efi): A nivel de unidades de paisaje, la Efí varió entre 65.0 a $77.4 \%$. A nivel de ecomosaicos en la matriz fue $86 \%$ mientras que para el bosque $89 \%$ (Cuadro 1).

Riqueza de especies: El total de especies de moluscos registrado fue de 50, mariposas 74 , aves 218 y árboles 172 . En total 514 especies fueron identificadas en el paisaje. La riqueza total dentro de las unidades de paisaje osciló desde las 147 especies en los potreros hasta las 279 especies en vegetación secundaria madura. A nivel de ecomosaicos la riqueza total registrada fue de 411 y 385 especies en la matriz y en el bosque, respectivamente. Con excepción del grupo de flora arbórea, el resto de grupos biológicos comparten el 50\% de las especies (Fig. 1). A nivel de grupos biológicos, las aves no mostraron diferencias ni a nivel de unidades de paisaje, ni entre mosaicos ( $p>0.05)$. La riqueza de árboles presentó diferencias entre unidades, mas no entre ecomosaicos. En el caso de los gasterópodos, la riqueza de PAD fue diferente al resto de unidades, entre ecomosaicos no hubo diferencias. Las mariposas mostraron diferencias entre unidades y ecomosaicos ( $<<0.05$; Cuadro 1).

Registros de especies: En lo general, los potreros y árboles en línea mostraron mayores diferencias en el número de individuos registrados en todos los grupos biológicos $(\mathrm{p}<0.05)$. A nivel de ecomosaicos, sólo las aves no mostraron diferencias (Cuadro 1).

Índices ecológicos: En las unidades del paisaje y con base en los valores promedio, la diversidad del índice Shannon-Wiener fluctuó entre 2.55 en unidades de potreros con árboles 
CUADRO 1

Índices de diversidad en unidades y ecomosaicos del paisaje estudiado

TABLE 1

Richness and diversity indices for landscape units and ecomosaics

\begin{tabular}{|c|c|c|c|c|c|c|c|c|c|c|c|}
\hline & \multirow{2}{*}{ GB } & \multicolumn{8}{|c|}{ Unidades de paisaje } & \multicolumn{2}{|c|}{ Ecomosaico } \\
\hline & & PAD & $\mathrm{AL}$ & $\mathrm{AJ}$ & $\mathrm{AM}$ & $\mathrm{RE}_{\mathrm{AM}}$ & $\mathrm{RE}_{\mathrm{AJ}}$ & $\mathrm{RE}_{\mathrm{PAD}}$ & REL & MA & $\mathrm{RE}$ \\
\hline \multirow[t]{5}{*}{ S obs. } & Árb & $18 \mathrm{~d}$ & $57 \mathrm{c}$ & $74 \mathrm{bc}$ & $92 \mathrm{a}$ & $88 \mathrm{a}$ & $86 a$ & $83 a b$ & $83 \mathrm{ab}$ & $128 \mathrm{a}$ & $124 \mathrm{a}$ \\
\hline & Aves & $85 a$ & $75 a$ & $108 \mathrm{a}$ & $112 \mathrm{a}$ & $117 \mathrm{a}$ & $111 \mathrm{a}$ & $114 \mathrm{a}$ & $98 \mathrm{a}$ & $173 a$ & $169 \mathrm{a}$ \\
\hline & Marip & $30 \mathrm{ab}$ & $33 a b$ & $49 a$ & $40 \mathrm{a}$ & $36 a b$ & $34 a b$ & $27 b$ & $36 a b$ & $65 \mathrm{a}$ & $51 b$ \\
\hline & Gast & $14 \mathrm{~b}$ & $33 \mathrm{a}$ & $34 \mathrm{a}$ & $35 a$ & $30 \mathrm{a}$ & $32 \mathrm{a}$ & $31 \mathrm{a}$ & $30 \mathrm{a}$ & $45 a$ & $41 \mathrm{a}$ \\
\hline & Total & 147 & 198 & 265 & 279 & 271 & 263 & 255 & 247 & 411 & 385 \\
\hline \multirow[t]{5}{*}{ S esp. } & Árb & 55 & 118 & 152 & 150 & 126 & 114 & 118 & 123 & 167 & 141 \\
\hline & Aves & 104 & 94 & 135 & 142 & 166 & 142 & 151 & 133 & 193 & 191 \\
\hline & Marip & 37 & 43 & 61 & 53 & 50 & 45 & 32 & 50 & 75 & 62 \\
\hline & Gast & 30 & 48 & 43 & 41 & 35 & 39 & 38 & 35 & 42 & 39 \\
\hline & Total & 226 & 303 & 291 & 386 & 377 & 340 & 339 & 341 & 477 & 433 \\
\hline Efi. & $\%$ & 65.0 & 65.4 & 67.8 & 72.3 & 71.9 & 77.4 & 75.2 & 72.4 & 86.2 & 88.9 \\
\hline \multirow[t]{4}{*}{ Ind. Reg. } & Árb & $38 \mathrm{~b}$ & $214 b$ & $617 \mathrm{a}$ & $675 a$ & $687 \mathrm{a}$ & $736 a$ & $653 a$ & $501 \mathrm{a}$ & $1544 b$ & $2577 \mathrm{a}$ \\
\hline & Aves & $1077 \mathrm{a}$ & $693 b$ & $743 b$ & $659 b$ & $469 b$ & $561 \mathrm{~b}$ & $571 b$ & $449 b$ & $3172 a$ & $2050 \mathrm{a}$ \\
\hline & Marip & $461 \mathrm{a}$ & $523 a$ & $454 a$ & $228 b$ & $176 b$ & $193 b$ & $176 b$ & $194 b$ & $1666 a$ & $739 b$ \\
\hline & Gast & $28 \mathrm{c}$ & $162 b$ & $238 \mathrm{ab}$ & $291 \mathrm{a}$ & $255 \mathrm{ab}$ & $267 a b$ & $239 a b$ & $302 a$ & $719 b$ & $1063 a$ \\
\hline \multirow[t]{4}{*}{$\mathrm{H}^{\prime}$} & Árb & 2.66 & 3.43 & 3.21 & 3.68 & 3.74 & 3.84 & 3.73 & 3.58 & 3.86 & 3.93 \\
\hline & Aves & 3.16 & 3.50 & 4.07 & 4.17 & 4.33 & 4.23 & 4.28 & 4.1 & 4.09 & 4.46 \\
\hline & Marip & 1.94 & 1.90 & 2.85 & 3.06 & 2.95 & 2.85 & 2.81 & 2.79 & 2.90 & 3.04 \\
\hline & Gast & 2.43 & 2.94 & 3.04 & 3.17 & 2.98 & 2.96 & 2.95 & 2.94 & 3.18 & 3.03 \\
\hline \multirow[t]{4}{*}{ D } & Árb & 0.09 & 0.06 & 0.09 & 0.05 & 0.04 & 0.03 & 0.04 & 0.05 & 0.05 & 0.03 \\
\hline & Aves & 0.09 & 0.05 & 0.03 & 0.02 & 0.02 & 0.02 & 0.02 & 0.03 & 0.03 & 0.02 \\
\hline & Marip & 0.29 & 0.29 & 0.11 & 0.07 & 0.08 & 0.09 & 0.08 & 0.10 & 0.11 & 0.07 \\
\hline & Gast & 0.11 & 0.07 & 0.06 & 0.05 & 0.07 & 0.07 & 0.07 & 0.07 & 0.06 & 0.07 \\
\hline \multirow[t]{4}{*}{$\mathrm{E}$} & Árb & 0.92 & 0.85 & 0.75 & 0.81 & 0.84 & 0.86 & 0.84 & 0.81 & 0.80 & 0.82 \\
\hline & Aves & 0.71 & 0.81 & 0.87 & 0.88 & 0.91 & 0.90 & 0.90 & 0.89 & 0.79 & 0.87 \\
\hline & Marip & 0.57 & 0.54 & 0.73 & 0.83 & 0.82 & 0.81 & 0.86 & 0.78 & 0.70 & 0.77 \\
\hline & Gast & 0.92 & 0.84 & 0.86 & 0.89 & 0.88 & 0.85 & 0.86 & 0.87 & 0.84 & 0.82 \\
\hline \multirow[t]{3}{*}{ Media } & $\mathrm{H}^{\prime}$ & 2.55 & 2.94 & 3.29 & 3.52 & 3.50 & 3.47 & 3.44 & 3.35 & 3.51 & 3.62 \\
\hline & D & 0.15 & 0.12 & 0.07 & 0.05 & 0.05 & 0.05 & 0.05 & 0.06 & 0.06 & 0.05 \\
\hline & E & 0.78 & 0.76 & 0.80 & 0.85 & 0.86 & 0.86 & 0.87 & 0.84 & 0.78 & 0.82 \\
\hline
\end{tabular}

AJ=vegetación secundaria, acahual o barbecho joven, AL=árboles en línea formando cercos vivos, AM=vegetación secundaria, acahual o barbecho maduro, MA=Matriz agropecuaria, $\mathrm{PAD}=$ potrero con árboles dispersos, $\mathrm{RE}=\mathrm{B}$ osque tropical lluvioso, REAJ=bosque referente de vegetación secundaria joven en ladera, REAM=bosque referente de vegetación secundaria madura en cima de montaña, REL=perturbaciones lineales o caminos en el bosque, REPAD=bosque referente de potreros en partes planas. Letras diferentes indican categorías diferentes (Tukey $p<0.05$ ). S obs.=Riqueza observada, $\mathrm{S}$ esp.=Riqueza esperada, Ind. Reg.=Individuos registrados, $\mathrm{H}^{\prime}=$ Diversidad de Shannon-Weiner, $\mathrm{D}=$ Dominancia de Simpson, E=Equitatividad de Pielou, Media=Promedios de los índices por grupo, Efi.=Eficiencia en la detección $(\%)$. GB=Grupo Biológico: Arb=Árboles, Marip=Nymphalidae frugívoras, Gast=Gasterópodos, Total=Total de especies .

$\mathrm{AJ}=$ young secondary vegetation or young fallow, $\mathrm{AL}=$ trees in a linear arrangement forming live fences, $\mathrm{AM}=$ mature secondary vegetation or mature fallow, $\mathrm{MA}=$ agricultural matrix, $\mathrm{PAD}=$ pasture with scattered trees, $\mathrm{RE}=$ tropical rainforest, REAJ=young secondary, hillside reference vegetation, REAM=mature secondary, hill crest reference vegetation, REL=linear disturbance or paths in forests, REPAD=forest reference vegetation for pastures with scattered trees in flat land. Different letters indicate different categories (Tukey $\mathrm{p}<0.05$ ). S obs.=observed species richness, $\mathrm{S}$ esp.=expected species richness, Ind. Reg.=number of individuals, H'=Shannon-Weiner diversity, D=Simpson's dominance index, E=Pielou's evenness index, Media=group means, Efi.=detection efficiency. GB=biological group: Arb=trees, Marip=frugivorous Nymphalidae, Gast=gastropods, Total=Total number of species. 
Similitud Bray-Curtis

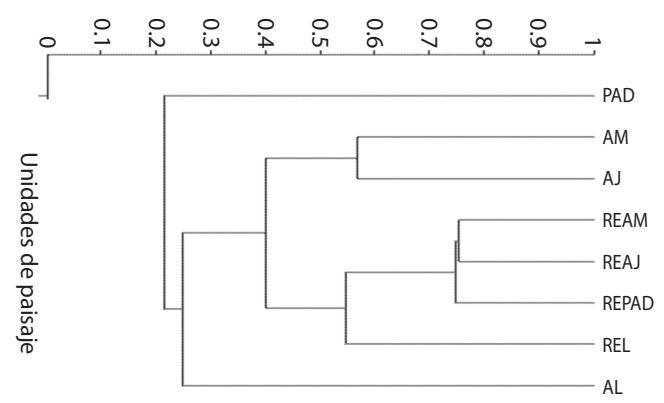

Arbóreas

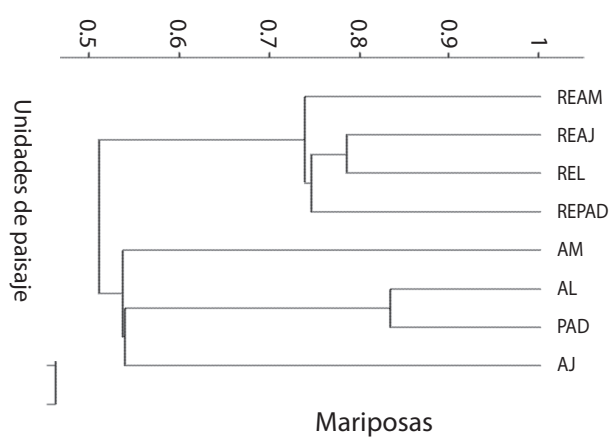

Similitud Bray-Curtis

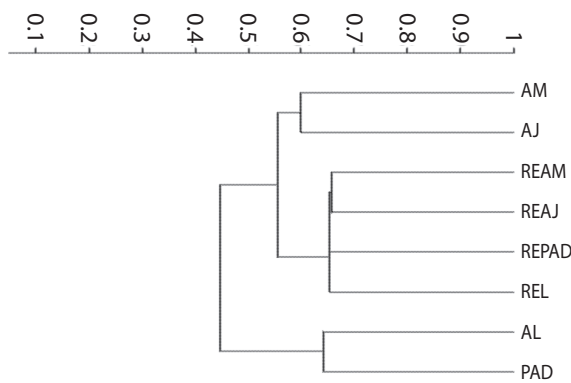

Aves
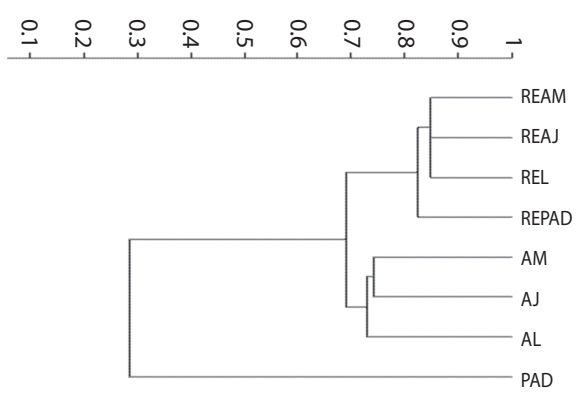

Gasterópodos

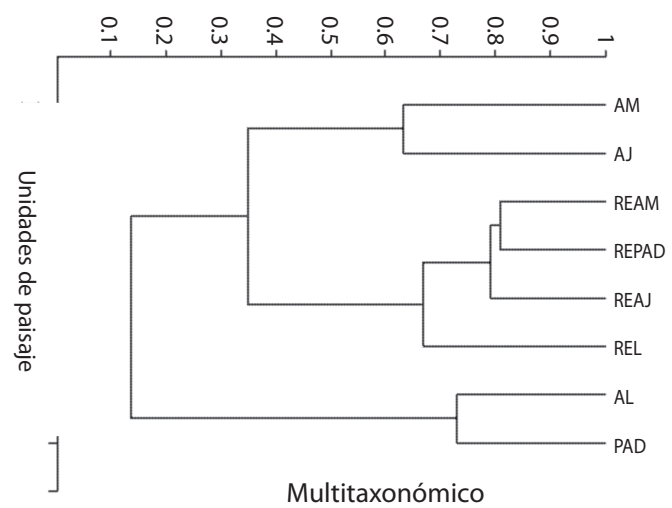

Fig. 1. Dendrogramas de similitud entre unidades de paisaje por grupo biológico y para el conjunto multitaxonómico. AJ y $\mathrm{AM}=$ vegetación secundaria joven y madura; $\mathrm{AL}$ y $\mathrm{PAD}=$ potreros con árboles en línea y dispersos; REAM=referente vegetación secundaria en la cima de montaña; REAJ=referente de vegetación secundaria joven en laderas; REPAD=referente de potreros con árboles dispersos; $y$ REL=referente de elementos lineales dentro del bosque perturbado

Fig. 1. Dendrograms of similarity between landscape units by biological group and multitaxa ensembles. AJ and AM=young and mature secondary vegetation; $\mathrm{AL}$ and $\mathrm{PAD}=$ pastureland with hedgerows and scattered trees; REAM=mature secondary vegetation reference community on hill top; REAJ = young secondary vegetation reference community on hillside; $\mathrm{REPAD}=$ reference vegetation for pastures with scattered trees in flat land; and REL=reference community for linear elements in disturbed forests. 
dispersos y 3.52 en acahuales maduros; el índice de Pielou mostró una distribución equitativa de especies con valores $\geq 0.76$, mientras que la dominancia de Simpson fue $\leq 0.15$, indicando que se trata de poblaciones con abundancias similares. Esto mismo se observa a nivel de ecomosaicos ya que los valores promedio del índice Shannon-Wiener fueron 3.51 y 3.62, la dominancia de Simpson fue 0.06 y 0.05 , y la equitatividad de Pielou 0.78 y 0.82 para la matriz y para el bosque respectivamente. Por grupos biológicos, el índice de ShannonWiener, fluctúa entre 2.90 para mariposas y 4.09 para aves en la matriz, mientras que en el bosque esta varía entre 3.03 para gasterópodos y 4.46 para aves. A nivel de unidades de paisaje, las mariposas frugívoras mostraron baja equitatividad y con especies dominantes en potreros con árboles dispersos y en los árboles en línea ( $\mathrm{E} \geq 0.57, \mathrm{D}=0.29$; Cuadro 1).

Ordenamiento de hábitats: Con base en los cuatro grupos biológicos, se distinguieron tres grandes tipos de hábitats: a) bosque tropical, b) vegetación secundaria $\mathrm{y}, \mathrm{c}$ ) hábitats abiertos y fuertemente perturbados que incluyen a los potreros con árboles dispersos y los árboles en línea. El primer tipo corresponde al ecomosaico de bosque, mientras que los dos últimos son parte de la matriz agropecuaria (Fig. 2).

El análisis de agrupamiento o conglomerados confirma la separación de los tres grandes tipos de hábitats arriba mencionados, tanto al analizar cada grupo biológico en particular, como cuando se incluyen todas las especies al hacer el análisis multitaxonómico (Fig. 1).

Especies indicadoras: Con excepción de los moluscos terrestres, todos los grupos biológicos mostraron al menos una especie característica para cada tipo de hábitat del paisaje (bosque, vegetación secundaria o potreros). Del total de especies, 107 (21\%) resultaron indicadoras de algún tipo de hábitat, 45 especies son árboles (26\%), 38 aves (18\%), 14 mariposas (18\%) y diez gasterópodos (20\%; Apéndice). De estas, 18 especies de árboles, 19 de aves, siete de mariposas y cinco de gasterópodos tienen un $\mathrm{InVal} \geq 70 \%$.

En los potreros, dos especies de árboles son características, Byrsonima crassifolia y Guazuma ulmifolia, de las cuales la primera es cultivada por su fruta y la segunda es conservada por su valor forrajero. Diez y ocho especies de aves resultaron características de este tipo de hábitat, destacando Sporophila americana, S. torqueola, Tyrannus verticalis y Crotophaga sulcirostris (InVal $\geq 85.2 \%, \mathrm{p}=0.001)$. Las mariposas Nymphalidae están representadas por siete especies, de las cuales destacan Hamadryas feronia farinulenta y $H$. laodamia saurites

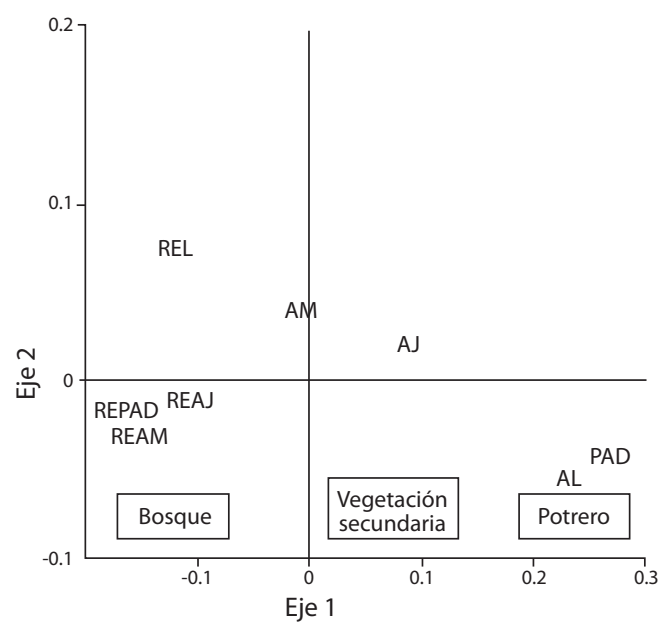

Fig. 2. Ordenamiento dimensional no métrico con base en múltiples táxones que separa los tres tipos de hábitats identificados. AJ y AM=vegetación secundaria joven y madura; $\mathrm{AL}$ y $\mathrm{PAD}=$ potreros con árboles en línea y dispersos; REAM=referente vegetación secundaria en la cima de montaña; REAJ=referente de vegetación secundaria joven en laderas; REPAD=referente de potreros con árboles dispersos; y REL=referente de elementos lineales dentro del bosque perturbado.

Fig. 2. Non-metric multi-dimensional ordination based on multiple taxa that distinguish among the three habitats types identified. $\mathrm{AJ}$ and $\mathrm{AM}=\mathrm{young}$ and mature secondary vegetation; $\mathrm{AL}$ and $\mathrm{PAD}=$ pastureland with hedgerows and scattered trees; REAM=mature secondary vegetation reference community on hill top; REAJ=young secondary vegetation reference community on hillside; $\mathrm{REPAD}=$ reference community for pastureland with dispersed trees; and REL=reference community for linear elements in disturbed forests. 
(InVal $\geq 83.7 \%, p=0.001$ respectivamente). Ninguna especie de molusco es indicadora de este tipo de hábitat.

En la vegetación secundaria, ocho especies de árboles son características de estas unidades del paisaje, sobresaliendo Heliocarpus donnell-smithii y Trichospermun mexicanun (InVal=90.1\% y $77.6 \%, \mathrm{p}=0.001$ respectivamente). Diez especies de aves estuvieron asociadas con este hábitat, destacándose Leptotila verreauxii y Ortalis vetula (InVal $\geq 80 \%$, $\mathrm{p}=0.001$ ). Las mariposas frugívoras sólo fueron moderadamente características de acahuales, de las tres especies registradas como indicadoras, los biblídinos Catonephele mexicana y Hamadryas amphinome mexicana alcanzaron un InVal $\geq 61 \%$ (p $\leq 0.005)$. De los gasterópodos, solamente se registraron dos especies, Lucidela lirata y Bulimulus corneus (InVal $=63.3 \%$ y $60.7 \%, \mathrm{p} \leq 0.017$ respectivamente).

En el bosque tropical lluvioso, como vegetación nativa de referencia, se identificaron 35 especies de árboles características entre las que destacan Chionanthus oblanceolatus, Rinorea guatemalense y Pouteria campechiana (InVal $\geq 90 \%, \mathrm{p}=0.001$ ). Las aves de bosque sumaron diez especies, y las más importantes (InVal $\geq 83 \%, \mathrm{p}=0.001$ ) fueron las insectívoras Lipaugus unirufus y Schiffornis turdinus comunes en el sotobosque herbáceo y Pipra mentalis que frecuenta el estrato arbóreo bajo, seguidos por el granívoro-frugívoro del dosel Ramphastos sulfuratus (InVal=81.5\%, $\mathrm{p}=0.001$ ). Hubo cuatro mariposas características de bosque, las Ithominae Oleria victorine paula y Napeogenes tolosa tolosa, la Morphinae Caligo uranus y la Satyrinae Taygetis virgilia (InVal $\geq 67.5 \%, \mathrm{p} \leq 0.004$ ). El grupo de especies más sensible por su escasa movilidad, el de los gasterópodos terrestres, que aportó ocho especies como indicadoras de bosque, sobresaliendo Carychium exiguum mexicanus y Helicina oweniana (InVal=82.6 y $80.9 \%$, $\mathrm{p}=0.001$ respectivamente).

Correlaciones entre especies. En el bosque, las correlaciones mostraron que los gasterópodos Carychium exiguum mexicanus,
Coelocentrum turris, Glyphyalinia aff. indentata y Helicina oweniana se relacionan significativamente (Pearson, $\mathrm{p}<0.05$ ) con todas las especies indicadoras de la fauna. No obstante, la relación entre la malacofauna y la vegetación arbórea del bosque es menos consistente. Por ejemplo, Glyphyalinia aff. indentata se correlacionó con 29 especies de árboles, Helicina oweniana se correlacionó con 23, mientras Carychium exiguum mexicanus lo hizo con 18 de las 35 especies de árboles de bosque. A pesar de esto, en conjunto estas tres especies se correlacionaron con todas las especies de todos los grupos biológicos característicos del bosque, excepto por tres árboles (Aspidosperma cruentum, Eugenia sp. y Guarea glabra). En particular Glyphyalinia aff. indentata (Zonitidae) se correlaciona con $90 \%$ (51) de todas las especies indicadoras del bosque, con la excepción de seis especies de árboles (Aspidosperma cruentum, Eugenia sp., Guarea glabra, Miconia argentea, Pouteria sp. 1 y Simira salvadorensis; Apéndice).

\section{DISCUSIÓN}

Eficiencia de muestreo: La eficiencia de captura de los múltiples taxones mediante el método combinado de muestreo aplicado, es similar a la que encuentran Manley et al. (2005), en su propuesta para la evaluación y monitoreo en campo de la biodiversidad a escala ecorregional (89\%). Esto, da apoyo a la utilidad de los métodos de muestreo aplicados en este estudio, con la particularidad y ventaja de que funcionan bajo condiciones neotropicales.

La diversidad en las unidades y ecomosaicos del paisaje es de media a alta (sensu Ramírez 2006), pues en la mayoría de los casos el índice de Shannon-Wiener fluctúa entre 2.5 y 3.9 , valores que son frecuentes en ambientes poco alterados o procedentes de matrices agropecuarias que presentan una estructura heterogénea (Pérez et al. 2007). La riqueza de especies, no obstante, se incrementa en la matriz, contrario a los resultados que Schulze et al. (2004) observaron en un paisaje similar en Indonesia, pues en el paisaje estudiado 
la vegetación secundaria madura alcanza la mayor riqueza, superando a la del bosque. Pero al igual que Schulze et al. (2004) encontramos que las áreas más abiertas por efecto de la dinámica agropecuaria (potreros) son la de menor riqueza. En la matriz se ha perdido o está ausente aproximadamente la mitad de las especies que componen el referente original, lo que se ha encontrado en estudios similares en paisajes dominados por humanos en el Neotrópico (Daily et al. 2001, Pérez et al. 2006, Tobar \& Ibrahim 2010), pero a diferencia de los resultados encontrados por estos autores, algunos elementos de la matriz aportan tanta riqueza y abundancia de especies como la que constituye la estructura de especies nativas de referencia, que habita el bosque original (Ochoa-Gaona et al. 2007).

El arreglo espacial - y de manera implícita temporal - de las unidades del paisaje se presenta en un continuo de disturbio antropogénico, en el que la vegetación secundaria representa una fase intermedia que propicia alta riqueza, y en donde se alternan especies de hábitats perturbados y especies propias del bosque (OchoaGaona et al. 2007), esto, corresponde con lo descrito por el modelo de disturbio intermedio de Connell (1978). Por otra parte, aunque el cambio en la composición de especies es una evidencia del impacto negativo en la diversidad original de los bosques (González-Espinosa et al. 2004), la desaparición de esta fase de vegetación secundaria y la pérdida de remanentes de bosque nativo haría más homogéneo el paisaje y la pérdida de especies nativas del bosque sería permanente.

La conservación biológica y la producción agropecuaria en el trópico solo pueden compatibilizarse, como en el paisaje estudiado, si se mantiene un balance entre áreas boscosas conservadas y otras aprovechadas para las actividades económicas. La permanencia de áreas continuas y extensas de bosque tropical, conectadas a fragmentos dispersos del bosque, inmersos en una matriz agropecuaria heterogénea, dominada por sistemas agroforestales facilita el flujo biológico (Harvey \& Ibrahim 2003). Estos arreglos varían tanto en lo espacial como en lo temporal, pero su persistencia a través del paisaje dominado por humanos, mantiene la resiliencia de las comunidades biológicas propias de los ecosistemas boscosos por el flujo biológico desde los hábitats conservados hacia los que componen la matriz del paisaje. Ésta, a su vez es permeable debido a la composición y estructura arbórea característicos de las unidades del paisaje agropecuario del ejido, permitiendo su persistencia y su capacidad de recuperación (Folke et al. 2004).

Ordenamiento y agrupación: $\mathrm{Al}$ ordenar las unidades del paisaje, se comprobó que el bosque es el refugio de un conjunto de organismos relativamente característicos de ésta condición, y que constituyen la comunidad biótica de referencia para el bosque tropical lluvioso de la zona montañosa del paisaje tropical estudiado. La distinción de tres grupos de hábitats basada en la composición multitaxonómica, y que separa al bosque de la vegetación secundaria y, a ambas de los potreros (unidades PAD y AL), fue previamente detectada por Schulze et al. (2004), Sánchez et al. (2005), Harvey \& González-Villalobos (2007) y Pérez et al. (2007). De manera similar a nuestros resultados, estos autores mencionan que la diversidad es modificada por el cambio de uso forestal al agropecuario, y que el bosque mantiene una porción de especies características del mismo. Asimismo, concuerdan con nosotros en que el manejo de paisajes heterogéneos, con remanentes de bosque de tamaño suficiente para albergar a las especies propias del mismo, junto a prácticas de agricultura y ganadería que produzcan matrices heterogéneas, permiten una alta conservación de la biodiversidad y funcionalidad ecológica de los paisajes dominados por humanos.

Indicadores multitaxonómicos: Nuestros resultados muestran un patrón de mayor a menor diversidad de especies características en la secuencia bosque-vegetación secundaria-potreros (Apéndice). De forma similar a nuestros resultados, Schulze et al. (2004) 
encontraron que una proporción del 20\% de los organismos resultó buen predictor para los hábitats. Al aplicar un $\mathrm{InVal} \geq 70 \%, 49$ especies serían las más consistentes y estables como indicadoras ecológicas (McGeoch et al. 2002). Las especies de árboles características de bosque reportadas por Ochoa-Gaona et al. (2007): Brosimum alicastrum, Chionanthus oblanceolatus, Dialium guianensis, Pouteria campechiana, Protium copal, Quararibea funebris y Rinorea guatemalensis en nuestro estudio obtuvieron un $\mathrm{InVal} \geq 70 \%$. Las más importantes como indicadoras de éste hábitat por presentar InVal $\geq 90 \%$ resultaron ser: $C$. oblanceolatus, $R$. guatemalensis y $P$. campechiana. En la matriz agropecuaria nuestros hallazgos coinciden con las especies de árboles encontradas por Ferguson et al. (2003), tales como Bursera simaruba, Acacia mayana y Cecropia obtusifolia en vegetación secundaria y Guazuma ulmifolia en los potreros. En cuanto a aves, Arriaga-Weiss et al. (2008) también mencionan como especialistas de bosque a Lipaugus unirufus y Pipra mentalis que coinciden con nuestros registros, con más de $83 \%$ de InVal, mientras que en potreros mencionan a Crotophaga sulcirostris, Quiscalus mexicanus, Sporophila torqueola que en nuestros registros tienen un InVal $\geq 75 \%$. Por el contrario, estos mismos autores registran a Chondrohierax uncinatus y Myiodinastes luteiventris como especialistas de bosque, mientras que en nuestro estudio resultaron indicadoras de potreros con InVal $>73 \%$. De las especies de mariposas, DeVries \& Walla (2001) mencionan a Taygetis virgilia como característica de bosques del Neotrópico, lo cual se confirma con el resultado de este estudio con un InVal=67\%. En cuanto a moluscos, Snodgrass (1998) basado en registros paleontológicos - menciona a Carychium exiguum como una especie indicadora del bosque conservado y muy sensible a la perturbación; en nuestro trabajo, esta especie resultó característica de bosques con InVal $=82 \%$, que es el mayor valor de todos los moluscos. En los potreros, los árboles, aves y mariposas estuvieron bien representados, pero no así los moluscos, lo cual puede deberse a su restringida movilidad y a su sensibilidad a la insolación y menor humedad en este hábitat (Pérez et al. 2007), siendo por ello este grupo el más afectado por la pérdida de la cobertura del bosque tropical.

Cuatro especies de moluscos se correlacionaron con casi la totalidad de las especies pertenecientes a los otros grupos biológicos característicos del bosque. Esto es similar a lo reportado por Lawton et al. (1998), quienes encontraron que de ocho grupos biológicos correlacionados con base en su riqueza de especies, ninguno resultó buen predictor de la diversidad en general, por tanto, el uso de registros de individuos utilizado en ésta investigación resulta valioso metodológicamente. Estas cuatro especies podrían considerarse como predictoras de la diversidad, incluso mejor que los invertebrados indicadores reportados por Kerr et al. (2000), debido a que son más fáciles de muestrear e identificar. Esto, además responde a los señalamientos de Lindenmayer et al. (2000), quienes mencionan que aún no se han establecido las relaciones de posibles indicadores con la biodiversidad total. En este sentido, los gasterópodos se muestran como un grupo de especies indicadoras, tan efectivo como el de las aves mencionadas por Bani et al. (2006). A diferencia de estos autores, en nuestro estudio se correlacionaron todas las especies previamente identificadas como características del bosque, incorporando todos los grupos biológicos. Con este método, se detectaron las especies que posiblemente funcionan como indicadoras de biodiversidad del bosque tropical lluvioso estudiado, siendo estas las cuatro especies arriba mencionadas. Además, los gasterópodos por su sensibilidad a los impactos (Pérez et al. 2007) y su escasa vagilidad, constituyeron el grupo de organismos más relevantes como indicadores de la calidad del hábitat del bosque tropical lluvioso local.

Nuestros hallazgos confirman que los diferentes grupos biológicos contienen especies características de los diferentes tipos de hábitats del paisaje y en particular del bosque conservado. El aporte de este estudio, es el de identificar las especies de gasterópodos que resultaron indicadoras de la biodiversidad 
del bosque tropical lluvioso de la zona. Esto brinda una nueva perspectiva sobre el papel de especies sensibles de la mesofauna en el enriquecimiento de opciones para el monitoreo de la calidad ecológica de los bosques y su biodiversidad.

\section{AGRADECIMIENTOS}

A los ejidatarios de Niños Héroes de Chapultepec, Tenosique por su colaboración. A Isidro Pérez Hernández, Vicente López Moreno, Orlando Lara López y Eduardo Cambranis González por su apoyo en el trabajo de campo. Por su ayuda en la identificación de especies, a Miguel Martínez Icó y Ángeles Guadarrama (árboles), Jaqueline Gamboa Aguilar (gasterópodos) y Noemí Salas Suárez (mariposas). FOMIX-CONACYT-Tabasco, financió parcialmente este trabajo bajo el proyecto: "Especies forestales de uso múltiple: caracterización de sus usos y potencial de aplicación en planes de fomento forestal, agroforestal y silvopastoril en el municipio de Tenosique, Tabasco" TAB2007-C09-74820. A ECOSUR por las facilidades humanas, científicas, de infraestructura y logísticas. A la DIA-SRE de México por la beca 811-06-3-728.5/6457/07 para los estudios de doctorado del primer autor.

\section{RESUMEN}

El uso de especies indicadoras para caracterizar unidades ecológicas específicas es de gran importancia en la biología de la conservación/restauración. El objetivo del estudio fue identificar desde una perspectiva multitaxonómica, las especies que caracterizan distintas unidades de un paisaje. Así, se diferenciaron dos ecomosaicos: bosque tropical lluvioso y matriz agropecuaria con cuatro unidades de paisaje cada uno. Se incluyeron cuatro grupos biológicos: aves (muy móviles), mariposas frugívoras diurnas (moderadamente móviles), gasterópodos terrestres (poco móviles) y árboles (sésiles). La eficiencia de muestreo en los ecomosaicos fue $\geq 86 \%$. Se registraron 50 especies de moluscos, 74 de mariposas, 218 de aves y 172 de árboles, totalizando 514 especies. Mediante ordenamiento y agrupamiento, se diferenciaron tres tipos de hábitats: bosque tropical lluvioso, vegetación secundaria y potreros con árboles. Aplicando el método InVal $(\geq 50 \%)$, se identificaron 107 especies indicadoras, de las cuales 45 fueron árboles, 38 aves, 14 mariposas y diez gasterópodos. De éstas, 35 especies de árboles, diez de aves, cuatro de mariposas y ocho de gasterópodos son indicadoras del bosque. Diez, veintiocho, diez y dos especies (de cada grupo respectivamente) caracterizaron a la matriz agropecuaria. En el bosque, los gasterópodos Carychium exiguum, Coelocentrum turris, Glyphyalinia aff. indentata y Helicina oweniana se correlacionaron significativamente $(\mathrm{p}<0.05)$ con $90 \%$ de las especies indicadoras. Estos gasterópodos pueden, además de diferenciar la calidad del hábitat, ser indicadoras de la biodiversidad del bosque. La vegetación secundaria representó una fase intermedia de perturbación que propicia alta riqueza en la matriz agropecuaria. De manera que se aporta una perspectiva multitaxonómica que incluye el papel de la mesofauna en el monitoreo ecológico de agropaisajes.

Palabras clave: especies características, bosque lluvioso, paisajes culturales, biodiversidad, grupos de hábitat, México.

\section{REFERENCIAS}

Arriaga-Weiss, S., S. Calmé \& C. Kampichler. 2008. Bird communities in rainforest fragment: guild responses to habitat variables in Tabasco, Mexico. Biodivers. Conserv. 17: 173-190.

Bani, L., D. Massimino, L. Bottoni \& R. Massa. 2006. A multiscale method for selecting indicator species and priority conservation areas: a case study for broadleaved forests in Lombardy, Italy. Conserv. Biol. 20: 512-526.

Baqueiro-Cárdenas, E.R., L. Borabe, C.G. Goldaracena-Islas \& J. Rodríguez-Navarro. 2007. Los moluscos y la contaminación. Una revisión. Rev. Mex. Biodiv. 78: 1-7.

Beever, E.A., R.K. Swihart \& B.T. Bestelmeyer. 2006. Linking the concept of scale to studies of biological diversity: evolving approaches and tools. Divers. Distrib. 12: 229-235.

Berger, B. \& R. Dallinger. 1993. Terrestrial snails as quantitative indicators of environmental metal pollution. Environ. Monit. Assess. 25: 65-84.

Bibby, C., M. Jones \& S. Marsden. 1998. Expedition field techniques: bird surveys. Royal Geographical Society, Londres, Inglaterra.

Bustamante, R. \& A.A. Grez. 1995. Consecuencias ecológicas de la fragmentación de los bosques nativos. Ambient. Desarro. 11: 58-63.

Castellanos, P.M. \& C. Serrato. 2008. Diversidad de invertebrados acuáticos en un nacimiento de río en el 
páramo de Santurbán, norte de Santander. Rev. Acad. Colomb. Cienc. 32: 79-86.

Castellón, T.D. \& K.E. Sieving. 2006. An experimental test of matrix permeability and corridor use by an endemic understory bird. Conservat. Biol. 20: 135-145.

Chazdon, R.L. 2008. Beyond deforestation: restoring forest and ecosystems services on degraded land. Science 20: $1458-1460$.

Chazdon, R.L., C. Harvey, O. Komar, M. van Breugel, B.G. Ferguson, D.M. Griffith, M. Martínez-Ramos, H. Morales, R. Nigh, L. Soto-Pinto \& S.M. Philpot. 2008. Beyond reserves: A research agenda for conserving biodiversity in tropical human-modified landscapes. Biotropica 41: 142-153.

CITES. 1973. Convención sobre el Comercio Internacional de Especies Amenazadas de Flora y Fauna Silvestres. Washington DC, Washington, EEUU. (Consultado: 4 Noviembre 2010, www.cites.es/citesapp/ Legislacion+CITES/LegislacionAplicacion.htm).

Colwell, R.K. 2008. EstimateS: Statistical Estimation of Species Richness and Shared Species from samples. Version 8. University of Connecticut. (Disponible en: http://www.viceroy.eeb.uconn.edu/estimates).

Connell, J.H. 1978. Diversity in tropical rain forest and coral reefs. Science 199: 1302-1310.

Cook, W.M., R.M Anderson \& E.W. Schweiger. 2004. Is the matrix really inhospitable? Vole runway distribution in an experimentally fragmented landscape. Oikos 104: 5-14.

COP 10 - About the Conference of the Parties. (Consultado: 19 Noviembre 2010, www.cbd.int/cop10/about/).

Daily, G.C., P.R. Ehrlich \& G.A. Sánchez-Azofeifa. 2001. Countryside biogeography: use of human-dominated habitats by the avifauna of southern Costa Rica. Ecol. Appl. 11: 1-13.

DeVries, P.J. \& T.R. Walla. 2001. Species diversity and community structure in neotropical fruit-feeding butterflies. Biol. J. Linn. Soc. 74: 1-15.

Diario Oficial de la Federación. 2008. Decreto por el que se declara área natural protegida con la categoría de área de protección de flora y fauna, la región conocida como Cañón del Usumacinta, localizada en el Municipio de Tenosique, en el Estado de Tabasco, 22 de Septiembre de 2008. (Consultado: 29 junio 2010, vlex.com.mx/vid/categoria-flora-fauna-usumacintatenosique-42835362).
Dobson, A.P., A.D. Bradshaw \& A.J.M. Baker. 1997. Hopes for future: restoration ecology and conservation biology. Science 277: 515-522.

Driscoll, D.A. 2005. Is the matrix a sea? Habitat specificity in a naturally fragmented landscape. Ecol. Entomol. 30: 8-16.

Dufrene, M. \& P. Legendre. 1997. Species assemblages and indicator species: The need for a flexible asymmetrical approach. Ecol. Monogr. 67: 345-366.

Ellenberg, H. \& D. Mueller-Dombois. 1967. Tentative physiognomic-ecological classification of plant formations of the earth. Ber. Geobot. Inst. ETH, Stiftg. Rübel. Zürich 37: 21-55.

Ferguson, B.G., J. Vandermeer, H. Morales \& D.M. Griffith. 2003. Post-agricultural succession in El Petén, Guatemala. Conserv. Biol. 17: 818-828.

Folke, C., S. Carpenter, B. Walker, M. Scheffer, T. Elmqvist, L. Gunderson \& C.S. Holling. 2004. Regime shifts, resilience, and biodiversity in ecosystem management. Annu. Rev. Ecol. Evol. S. 35: 557-581.

Forman, R. \& M. Godron. 1986. Landscape ecology. Wiley, Nueva York, EEUU.

Gobierno del Estado. 1997. Municipio de Tenosique. Secretaria de Desarrollo Social y Protección Ambiental, Gobierno del Estado de Tabasco. Villahermosa, Tabasco, México.

González-Espinosa, M., J.M. Rey-Benayas, M. RamírezMarcial, M.A. Huston \& D. Golicher. 2004. Tree diversity in the northern Neotropics: regional patterns in highly diverse Chiapas, Mexico. Ecography 27: 741-756.

González-Valdivia, N., S. Ochoa-Gaona, E. Cambranis, O. Lara, I. Pérez-Hernández, A. Ponce-Mendoza, J.L. Rangel-Ruíz, J. Gamboa-Aguilar, C. Kampichler, C. Pozo y B.G. Ferguson. 2010. Gasterópodos terrestres asociados a un paisaje agropecuario y a un referente ecológico en el sureste de México. In L.J. RangelRuiz, J. Gamboa-Aguilar, S.L. Arriaga-Weiss \& W.M. Contreras-Sánchez (eds.). Perspectivas en Malacología Mexicana. Colección José N. Rovirosa. Universidad Juárez Autónoma de Tabasco. pp. 90-122.

Hammer, Ø., D. Harper \& P. Ryan. 2001. PAST: Paleontological Statistics Software Package for education and data analysis. Paleontol. Electron. 4: 1-9. (Disponible en línea: palaeo-electronica.org/2001_1/past/ issue1_01.htm).

Harvey, C. \& J. González-Villalobos. 2007. Agroforestry systems conserve species-rich but modified 
assemblages of tropical birds and bats. Biodivers. Conserv. 16: 2257-2292.

Harvey, C.A. \& M. Ibrahim. 2003. Diseño y manejo de la cobertura arbórea en fincas ganaderas para mejorar las funciones productivas y brindar servicios ecológicos. Agrof. en las Américas 10: 39-40.

Heino, J., K.T. Tolonen, J. Kotanen \& L. Paasivirta. 2009. Indicator groups and congruence of assemblage similarity, species richness and environmental relationships in littoral macroinvertebrates. Biodivers. Conserv. 18: 3085-3098.

Higgs, E. 2003. Nature for design: People, natural processes and ecological restoration. Massachusetts Institute of Technology, Cambridge, EEUU.

Holl, K.D. \& E.E. Crone. 2004. Applicability of landscape and island biogeography theory to restoration of riparian understorey plants. J. Appl. Ecol. 41: 922-933.

Holling, C.S. 2001. Understanding the complexity of economic, ecological and social systems. Ecosystems 4: 390-405.

Howell, S. \& S. Webb. 2005. A guide to the birds of México and northern Central America. Oxford, Oxford, Nueva York, EEUU.

Isaac-Márquez, R., B. de Jong, A. Eastmond, S. OchoaGaona \& S. Hernández. 2005. Estrategias productivas campesinas: un análisis de los factores condicionantes del uso de suelo en el Oriente de Tabasco, México. Universidad y Ciencia 21: 56-72.

Jobin, B., J. Beaulieu, M. Grenier, L. Bélanger, C. Maisonneuve, D. Bordage \& B. Filion. 2003. Landscape changes and ecological studies in agricultural regions, Québec, Canada. Landsc. Ecol. 18: 575-590.

Kattan, G.H., H. Álvarez-López \& M. Giraldo. 1994. Forest fragmentation and bird extinctions: San Antonio eighty years later. Conserv. Biol. 8: 138-146.

Kerr, J.T., A. Sugar \& L. Packer. 2000. Indicator taxa, rapid biodiversity assessment, and nestedness in an endangered ecosystem. Conserv. Biol. 14: 1726-1734.

Kupfer, J.A., G.P. Malanson \& S.B. Franklin. 2006. Not seeing the ocean for the islands: The mediating influence of matrix-based processes on forest fragmentation effects. Global Ecol. Biogeogr. 15: 8-20.

Lawton, J.H., D.E. Bignell, B. Bolton, G.F. Bloemers, P. Eggleton, P.M. Hammond, M. Hodda, R.D. Holt, T.B. Larsen, N.A. Mawdsley, N.E. Stork, D.S. Srivastava \& A.D. Watt. 1998. Biodiversity inventories, indicator taxa and effects of habitat modification in tropical forest. Nature 391: 72-76.

Lindenmayer, D.B., C.R. Margules \& D.B. Botkin. 2000. Indicators of diversity for ecologically sustainable forest management. Conserv. Biol. 14: 941-950.

Manley, P.N., M.D. Schlesinger, J.K. Roth \& B. Van Horne. 2005. A field-based evaluation of a presence-absence protocol for monitoring ecoregional-scale biodiversity. J. Wildl. Manag. 69: 950-966.

Marín, L., J.L. León-Cortés \& C. Stefanescu. 2009. The effect of an agro-pasture landscape on diversity and migration patterns of frugivorous butterflies in Chiapas, Mexico. Biodivers. Conserv. 18: 919-934.

McCune, B. \& M.J. Mefford. 1999. Multivariate analysis of ecological data. Version 4.25. MjM Software, Gleneden Beach, Oregon, EEUU.

McGeoch, M.A., B.J. Van Rensburg \& A. Botes. 2002. The verification and application of bioindicators: a case of study of dung beetles in a savanna ecosystem. J. Appl. Ecol. 39: 661-672.

Metzger, J.P. 2006. How deal with non-obvious rules for biodiversity conservation in fragmented areas. Nat. Conservacao. 4: 125-137.

Moreno, C.E. 2001. Método para medir la biodiversidad. M\&T-Manuales y Tesis SEA. CYTED-ORCYT/ UNESCO-SEA, Zaragoza, España.

Murcia, C. 1995. Edge effects in fragments: implications for conservation. Tree 10: 58-62.

Noss, R.F. 1990. Indicators for monitoring biodiversity: a hierarchical approach. Conserv. Biol. 12: 822-835.

Ochoa-Gaona, S., F. Hernández-Vázquez, B.H.J. de Jong \& F. Gurri. 2007. Pérdida de diversidad florística ante un gradiente de intensificación del sistema agrícola de roza-tumba-quema: un estudio de caso en la Selva Lacandona, Chiapas, México. Bol. Soc. Bot. Mex. 81: $65-80$.

Ochoa-Gaona, S., I. Pérez-Hernández \& B. De Jong. 2008. Fenología reproductiva de las especies arbóreas del bosque tropical de Tenosique, Tabasco, México. Rev. Biol. Trop. 56: 657-673.

Ochoa-Gaona, S., M. González-Espinosa, J.A. Meave \& V. Sorani-Dal Bon. 2004. Effect of forest fragmentation on the woody flora of the highlands of Chiapas, Mexico. Biodivers. Conserv. 13: 867-884.

Pérez, A.M., M. Sotelo, F. Ramírez, A. López \& I. Siria. 2006. Conservación de la biodiversidad en sistemas 
silvopastoriles de Matiguás y Rio Blanco (Matagalpa, Nicaragua). Ecosistemas 15: 125-141.

Pérez, A.M., M. Sotelo, I. Siria, R. Alkemade \& L. Aburto. 2007. Developing a species-based model for biodiversity assessment in an agricultural landscape in Nicaragua. Gaia 8: 1-54.

Perfecto, I. \& J. Vandermeer. 2002. Quality of agroecological matrix in a tropical montane landscape: ants in coffee plantations in southern of Mexico. Conserv. Biol. 16: 174-182.

Perfecto, I. \& J. Vandermeer. 2008. Biodiversity conservation in tropical agroecosystems: A new conservation paradigm. Ann. NY Acad. Sci. 1134: 173-200.

Pozo, C., N. Salas-Suárez, B. Prado-Cuellar \& E. May-Uc. 2009. Riqueza de mariposas diurnas (Lepidóptera: Rophalocera) en el Santuario del Manatí y una propuesta para su uso en el monitoreo de ambientes terrestres del área, p. 139-147. In J. Espinoza-Avalos, G.A. Islebe \& H.A. Hernández-Arana (eds.). El sistema ecológico de la bahía de Chetumal/Corozal: costa occidental del Mar Caribe., ECOSUR, Chetumal, Quintana Roo, México.

Pozo, C., A. Luis-Martínez, J. Llorente-Bousquets, N. Salas-Suárez, A. Maya-Martínez, I. Vargas-Fernández \& A. Warren. 2008. Seasonality and phenology of the butterflies (Lepidoptera: Papilionoidea and Hesperioidea) of Mexico's Calakmul region. Fla. Entomol. 91: 407-422.

Ramírez, A. 2006. Ecología: Métodos de muestreo y análisis de poblaciones y comunidades. Editorial Pontificia Universidad Javeriana, Bogotá, Bogotá, Colombia.

Ramírez-Marcial, N., M. González-Espinosa \& G. Williams-Linera. 2001. Anthropogenic disturbance and tree diversity in montane rain forests in Chiapas, Mexico. Forest. Ecol. Manag. 154: 311-326.

Rangel-Ruiz, L.J. \& J. Gamboa-Aguilar. 2000. Gasterópodos epicontinentales de la Reserva de la Biósfera Pantanos de Centla, Tabasco. Universidad y Ciencia 15: $129-140$.

Rangel-Ruiz, L.J. \& J. Gamboa-Aguilar. 2006. Listado preliminar de gasterópodos terrestres de "Boca del Cerro" Tenosique, Tabasco, México. Kuxulkab’ 11: 51-57.

Ruiz-Díaz, F., F.J. Barón López, E. Sánchez Font \& L. Parras Guijosa. 1998. Bioestadística. Métodos y aplicaciones. Facultad de Medicina, Universidad de Málaga, España.
Rzedowski, J. 2006. Vegetación de México. 1ra. Edición digital, Comisión Nacional para el Conocimiento y Uso de la Biodiversidad, Distrito Federal, México. (Disponible en línea en http://www.conabio.gob. $\mathrm{mx} /$ institucion/centrodoc/doctos/vegetacion_de_ mexico.html).

Sánchez, D., C. Harvey, A. Grijalva, A. Medina, S. Vílchez \& B. Hernández. 2005. Diversidad, composición y estructura de la vegetación en un agropaisaje ganadero en Matiguás, Nicaragua. Rev. Biol. Trop. 53: 387-414.

Saunders, D.A., R.J. Hobbs \& C.R. Margules. 1991. Biological consequences of ecosystems fragmentation: a review. Conserv. Biol. 5: 18-32.

Schulze, C.H., M. Waltert, P.J.A. Kessler, R. Pitopang, R. Shahabuddin, D. Veddeler, M. Mühlenberg, S.R. Gradstein, C. Leuschner, I. Steffan-Dewenter \& T. Tscharntke. 2004. Biodiversity indicator groups of tropical land-use systems: comparing plants, birds, and insects. Ecol. Appl. 14: 1321-1333.

Secrest, M.F., M.R. Willig \& L.L. Peppers. 1996. The legacy of disturbance on habitat associations of terrestrial snail in the Luquillo Experimental Forest, Puerto Rico. Biotropica 28: 502-514.

Snodgrass, K.M. 1998. Theler ancient climate as inferred by land snails at the Brokenleg Bend locality, Oklahoma. J. Undergraduate Res. 1: 154-160.

Spellerberg, I.F. 2005. Monitoring ecological change. Universidad de Cambridge, Cambridge, Inglaterra.

Stouffer, P.C. \& R.O. Bierregaard. 1995. Use of Amazonian forest fragments by understory insectivorous birds. Ecology 76: 2429-2445.

Tejeda-Cruz, C., K. Mehltreter \& V.J. Sosa. 2008. Indicadores ecológicos multitaxonómicos, p. 271-278. In R.H. Manson, V. Hernández-Ortiz, S. Gallina \& K. Mehltreter (eds.). Agroecosistemas cafetaleros de Veracruz: Biodiversidad, manejo y conservación. Instituto de Ecología e Instituto Nacional de Ecología. México.

Tobar, D. \& M. Ibrahim. 2010. ¿Las cercas vivas ayudan a la conservación de la diversidad de mariposas en paisajes agropecuarios? Rev. Biol. Trop. 58: 447.

Tobar, D., M. Ibrahim \& F. Casasola. 2007. Diversidad de mariposas en un paisaje agropecuario del Pacífico Central de Costa Rica. Agrof. en las Américas 45: 58-65.

Toledo, V.M. \& N. Barrera-Bassols. 2008. La memoria biocultural: La importancia ecológica de las sabidurías tradicionales. Perspectivas Agroecológicas 3, Icaria, Barcelona, España. 
Turner, I.M. 1996. Species loss in fragments of tropical rain forest: a review of the evidence. J. Appl. Ecol. 33: 200-209.

Turner, M., R. Gardner \& R.O’Neill. 2001. Landscape ecology in theory and practice: pattern and process. Springer, Nueva York, EEUU.

Van Perlo, B. 2006. Birds of México and Central America. Illustrated Checklist. Princeton, Nueva Jersey, EEUU.

Vera-Ardila, M.L. \& E.L. Linares. 2005. Gastrópodos de la región subxerofítica de La Herrera, Mosquera, Cundinamarca, Colombia. Rev. Acad. Colomb. Cienc. 29: 439-456.

Villavicencio-Enríquez, L. \& J.I. Valdez-Hernández. 2003. Análisis de la estructura arbórea del sistema agroforestal rusticano de café en San Miguel, Veracruz, México. Agrociencia 37: 413-423.
Walker, B., S. Carpenter, J. Anderies, N. Abel, G. Cumming, M. Janssen, L. Lebel, J. Norberg, G.D. Petersen \& R. Pritchard. 2002. Resilience management in social-ecological systems: a working hypothesis for a participatory approach. Cons. Ecol. 6: 14.

Wilcox, B.A., D.D. Murphy, P.R. Ehrlich \& G.T. Austin. 1986. Insular biogeography of the montane butterfly faunas in the Great Basin: Comparison with birds and mammals. Oecologia 69: 188-194.

Young, T.P. 2000. Restoration ecology and conservation biology. Biol. Conserv. 92: 73-83.

Zonneveld, I. 1989. The land unit: A fundamental concept in landscape ecology, and its application. Landscape Ecol. 3: 67-83.

Zuur, A., E. Ieno \& G. Smith. 2007. Analyzing ecological data, p. 163-179. In M. Gail, K. Krickeberg, J. Samet, A. Tsiatis \& W. Wong (eds.). Statistics for biology and health. Springer, Nueva York, EEUU. 
APÉNDICE

Indicadores multitaxonómicos de los tipos de hábitats en que se agrupan las unidades del paisaje

\section{APPENDIX}

Multitaxa indicators of the habitats types into which the landscape units are grouped

\begin{tabular}{|c|c|c|c|c|}
\hline \multicolumn{5}{|c|}{ Especies características del bosque tropical lluvioso } \\
\hline Grupo biológico & Especie & Familia & InVal $(\%)$ & $\mathrm{p}$ \\
\hline Árbol & Alchornea latifolia Sw. & Euphorbiaceae & 56.3 & 0.008 \\
\hline Árbol & Aspidosperma cruentum Woodson & Apocynaceae & 57.1 & 0.002 \\
\hline Árbol & Aspidosperma megalocarpon Müll. Arg. & Apocynaceae & 74.1 & 0.001 \\
\hline Árbol & Brosimum alicastrum $\mathrm{Sw}$. & Moraceae & 87.6 & 0.001 \\
\hline Árbol & Brosimum lactescens (S. Moore) C.C. Berg. & Moraceae & 54.4 & 0.010 \\
\hline Árbol & Calophyllum brasiliense Cambess. & Clusiaceae & 55.2 & 0.004 \\
\hline Árbol & Casearia sylvestris $\mathrm{Sw} . *$ & Flacourtiaceae & 89.6 & 0.001 \\
\hline Árbol & Chionanthus oblanceolatus (B.L. Rob.) P.S. Green* & Oleacea & 100 & 0.001 \\
\hline Árbol & Chione chiapasensis Stand. & Rubiaceae & 64.3 & 0.002 \\
\hline Árbol & Cryosophila argentea Bartlett $^{\mathrm{NOM}}$ & Arecaceae & 81.9 & 0.001 \\
\hline Árbol & Dendropanax arboreus (L.) Decne. \& Planch. & Araliaceae & 56.6 & 0.009 \\
\hline Árbol & Dialium guianensis (Aubl.) Sandwith & Fabaceae & 70.0 & 0.001 \\
\hline Árbol & Eugenia sp. & Myrtaceae & 73.5 & 0.001 \\
\hline Árbol & Guarea glabra Vahl. & Meliaceae & 64.7 & 0.001 \\
\hline Árbol & Ilex costaricensis Donn. Sm. & Aquifoliaceae & 68.8 & 0.001 \\
\hline Árbol & Manilkara chicle (Pittier) Gilly & Sapotaceae & 71.4 & 0.001 \\
\hline Árbol & Manilkara zapota (L.) P. Royen & Sapotaceae & 83.6 & 0.001 \\
\hline Árbol & Miconia argentea $(\mathrm{Sw}.) \mathrm{DC}$ & Melastomataceae & 53.7 & 0.006 \\
\hline Árbol & Nectandra lundellii C.K. Allen & Lauraceae & 81.9 & 0.001 \\
\hline Árbol & Nectandra salicifolia (Kunth) Nees & Lauraceae & 59.1 & 0.001 \\
\hline Árbol & Ouratea lucens (Kunth) Engl. & Ochnaceae & 57.1 & 0.004 \\
\hline Árbol & Oxandra belicensis (Lundell) Lundell & Annonaceae & 64.3 & 0.001 \\
\hline Árbol & Posoqueria latifolia (Rudge) Roem. \& Schult. & Rubiaceae & 58.2 & 0.002 \\
\hline Árbol & Pouteria campechiana (Kunth) Baehni* & Sapotaceae & 90.8 & 0.001 \\
\hline Árbol & Pouteria reticulata (Eng.) Eymma & Sapotaceae & 83.6 & 0.001 \\
\hline Árbol & Pouteria sp. 1 & Sapotaceae & 54.2 & 0.005 \\
\hline Árbol & Pouteria sp. 2 & Sapotaceae & 64.3 & 0.001 \\
\hline Árbol & Protium copal (Schltdl. \& Cham.) Eng. & Anancardiaceae & 79.4 & 0.001 \\
\hline Árbol & Pseudolmedia oxyphyllaria Donn. Sm. & Moraceae & 87.0 & 0.001 \\
\hline Árbol & Psycothria chiapensis Standl. & Rubiaceae & 58.1 & 0.004 \\
\hline Árbol & Quararibea funebris (La Llave) Vischer & Bombacaceae & 87.7 & 0.001 \\
\hline Árbol & Rinorea guatemalensis (S. Watson) Bartlett* & Violaceae & 95.2 & 0.001 \\
\hline Árbol & Sebastiania tuerckheimiana (Pax \& K. Hoffm.) & Euphorbiaceae & 57.2 & 0.010 \\
\hline Árbol & Simira salvadorensis (Stand1.) Steyerm. & Rubiaceae & 55.2 & 0.006 \\
\hline Árbol & Trophis racemosa $(\mathrm{L}$.$) Urb.$ & Moraceae & 63.0 & 0.002 \\
\hline Aves & Dendrocincla anabatina Sclater ${ }^{\mathrm{NOM}}$ & Dendrocolaptidae & 50 & 0.016 \\
\hline Aves & Columba cayennensis Bonnaterre & Columbidae & 58.2 & 0.004 \\
\hline Aves & Geotrygon montana Linnaeus & Columbidae & 55.6 & 0.005 \\
\hline Aves & Lipaugus unirufus Sclater & Cotingidae & 88.5 & 0.001 \\
\hline Aves & Pipra mentalis Sclater & Pipridae & 83.3 & 0.001 \\
\hline Aves & Ramphastos sulfuratus Lesson & Ramphastidae & 81.5 & 0.001 \\
\hline
\end{tabular}


APÉNDICE (Continuación)

Indicadores multitaxonómicos de los tipos de hábitats en que se agrupan las unidades del paisaje

APPENDIX (Continued)

Multitaxa indicators of the habitats types into which the landscape units are grouped

\begin{tabular}{|c|c|c|c|c|}
\hline Grupo biológico & Especie & Familia & InVal & $\mathrm{p}$ \\
\hline Aves & Schiffornis turdinus (Wied-Neuwied) & Cotingidae & 83.3 & 0.001 \\
\hline Aves & Trogon massena Gould & Trogonidae & 61.1 & 0.001 \\
\hline Aves & Uropsila leucogastra (Gould) & Trogloditidae & 60.4 & 0.003 \\
\hline Aves & Xiphorhynchus flavigaster Swainson & Dendrocolaptidae & 64.5 & 0.002 \\
\hline Mariposas & Caligo uranus Herrich-Schäffer & Morphinae & 82.4 & 0.002 \\
\hline Mariposas & Napeogenes tolosa tolosa (Hewitson) & Ithomiinae & 72.7 & 0.001 \\
\hline Mariposas & Oleria victorine paula (Weymer) & Ithomiinae & 84.2 & 0.001 \\
\hline Mariposas & Taygetis virgilia (Cramer) & Satyrinae & 67.5 & 0.004 \\
\hline Gasterópodos & Carychium exiguum mexicanus Say & Carychiidae & 82.6 & 0.001 \\
\hline Gasterópodos & Coelocentrum turris Pfeiffer & Urocoptidae & 76.3 & 0.001 \\
\hline Gasterópodos & Glyphialinia aff. indentata & Zonitidae & 72.7 & 0.001 \\
\hline Gasterópodos & Helicina oweniana Pfeiffer & Helicinidae & 80.9 & 0.001 \\
\hline Gasterópodos & Miradiscops opal (Pilsbry) & Systrophiidae & 62.6 & 0.001 \\
\hline Gasterópodos & Neocyclotus dysonii ambiguum Martens & Cyclophoridae & 58.2 & 0.011 \\
\hline Gasterópodos & Spiraxis sp. 2 & Spiraxidae & 77.5 & 0.001 \\
\hline Gasterópodos & Thysanophora impura Morelet & Thysanophoridae & 51.0 & 0.007 \\
\hline \multicolumn{5}{|c|}{ Especies características de la vegetación secundaria } \\
\hline Grupo biológico & Especie & Familia & InVal & $\mathrm{p}$ \\
\hline Árbol & Acacia mayana Lundell & Fabaceae & 61.4 & 0.007 \\
\hline Árbol & Bursera simaruba (L.) Sarg. & Burseraceae & 55.9 & 0.003 \\
\hline Árbol & Cecropia obtusifolia Bertol & Cecropiaceae & 56.1 & 0.007 \\
\hline Árbol & Heliocarpus donnell-smithii Rose* & Tiliaceae & 90.1 & 0.001 \\
\hline Árbol & Lippia cardiostegia Benth. & Lamiaceae & 63.8 & 0.006 \\
\hline Árbol & Myriocarpa longipes Liebm. & Urticaceae & 53.8 & 0.005 \\
\hline Árbol & Redhera penninervia Stand. \& Moldenke & Verbenaceae & 67.6 & 0.005 \\
\hline Árbol & Trichospermum mexicanum (DC.) Baill. & Ulmaceae & 77.6 & 0.001 \\
\hline Aves & Arremonops chloronotus Salvin & Emberiziidae & 54.8 & 0.006 \\
\hline Aves & Cyanocorax morio Wagler & Corvidae & 64.3 & 0.002 \\
\hline Aves & Helmitheros vermivorus Gmelin & Emberiizidae & 64.5 & 0.001 \\
\hline Aves & Leptotila verreauxi Lawrence & Columbidae & 85.1 & 0.001 \\
\hline Aves & Ortalis vetula Wagler & Cracidae & 80.5 & 0.001 \\
\hline Aves & Ramphocaenus melanurus Vieillot & Sylviidae & 57.7 & 0.004 \\
\hline Aves & Thryothorus ludovicianus Vieillot & Trogloditidae & 52.5 & 0.016 \\
\hline Aves & Thryothorus modestus Cabanis & Trogloditidae & 55.8 & 0.005 \\
\hline Aves & Troglodytes musculus Vieillot & Trogloditidae & 62.7 & 0.001 \\
\hline Aves & Vermivora peregrina (A. Wilson) & Emberiizidae & 52.2 & 0.005 \\
\hline Mariposas & Catonephele mexicana (Jenkins \& R.G. Maza) & Biblidinae & 63.6 & 0.003 \\
\hline Mariposas & Hamadryas amphinome mexicana (Lucas) & Biblidinae & 61.0 & 0.005 \\
\hline Mariposas & Pteronymia cotytto cotytto (Guérin) & Ithomiinae & 53.9 & 0.021 \\
\hline Gasterópodos & Lucidela lirata (Pfeiffer) & Helicinidae & 63.3 & 0.001 \\
\hline Gasterópodos & Bulimulus corneus (Sowerby) & Bulimulidae & 60.7 & 0.017 \\
\hline
\end{tabular}


APÉNDICE (Continuación)

Indicadores multitaxonómicos de los tipos de hábitats en que se agrupan las unidades del paisaje

APPENDIX (Continued)

Multitaxa indicators of the habitats types into which the landscape units are grouped

\begin{tabular}{|c|c|c|c|c|}
\hline \multicolumn{5}{|c|}{ Especies características de potreros con árboles en línea y dispersos } \\
\hline Grupo biológico & Especie & Familia & InVal & $\mathrm{p}$ \\
\hline Árbol & Byrsonima crassifolia (L.) Kunth & Malphigiaceae & 62.5 & 0.001 \\
\hline Árbol & Guazuma ulmifolia Lam. & Sterculiaceae & 50.9 & 0.003 \\
\hline Aves & Bubulcus ibis Linnaeus & Ardeidae & 50 & 0.003 \\
\hline Aves & Chaetura vauxii Townsend & Apodidae & 50 & 0.001 \\
\hline Aves & Chondrohierax uncinatus Temminck ${ }^{\mathrm{NOM}}$ & Accipitridae & 73.5 & 0.001 \\
\hline Aves & Columba flavirostris Wagler & Columbidae & 59.6 & 0.015 \\
\hline Aves & Columbina minuta Linnaeus & Columbidae & 70.7 & 0.003 \\
\hline Aves & Crotophaga sulcirostrisSwainson & Cuculidae & 85.2 & 0.001 \\
\hline Aves & Dives dives Deppe & Icteriidae & 55 & 0.017 \\
\hline Aves & Myiarchus crinitus Linnaeus & Tyrannidae & 57.3 & 0.004 \\
\hline Aves & Myiodinastes luteiventris Sclater & Tyrannidae & 75.0 & 0.001 \\
\hline Aves & Myiozetetes similis Spix & Tyrannidae & 73.5 & 0.001 \\
\hline Aves & Pitangus suphuratus (Linnaeus) & Tyrannidae & 75.1 & 0.001 \\
\hline Aves & Quiscalus mexicanus Gmelin & Icteriidae & 75 & 0.001 \\
\hline Aves & Sporophila americana Gmelin* & Emberiizidae & 95.7 & 0.001 \\
\hline Aves & Sporophila torqueola Bonaparte & Emberiizidae & 88.7 & 0.001 \\
\hline Aves & Thraupis episcopus Linnaeus & Emberiizidae & 80.8 & 0.001 \\
\hline Aves & Tyrannus melancholicus Cabanis & Tyrannidae & 75.4 & 0.002 \\
\hline Aves & Tyrannus savanna Vieillot & Tyrannidae & 75.0 & 0.001 \\
\hline Aves & Tyrannus verticalis Say & Tyrannidae & 87.5 & 0.001 \\
\hline Mariposas & Adelpha iphicleola iphicleola (H.W. Bates) & Apaturinae & 70.3 & 0.002 \\
\hline Mariposas & Dynamine postverta mexicana d’Almeida & Biblidinae & 65.6 & 0.001 \\
\hline Mariposas & Hamadryas februa ferentina (Godart) & Biblidinae & 55.2 & 0.025 \\
\hline Mariposas & Hamadryas feronia farinulenta (Frühstorfer)* & Biblidinae & 90.2 & 0.001 \\
\hline Mariposas & Hamadryas guatemalena marmarice (Frühstorfer) & Biblidinae & 78.8 & 0.001 \\
\hline Mariposas & Hamadryas laodamia saurites (Frühstorfer) & Biblidinae & 83.7 & 0.001 \\
\hline Mariposas & Opsiphanes cassina fabricii (Boisduval) & Morphinae & 54.0 & 0.007 \\
\hline
\end{tabular}

InVal=Valor del indicador, $\mathrm{p}=$ probabilidad según la prueba Monte Carlo. NOM=especies enlistadas en la Norma Oficial Mexicana como amenazadas o en peligro de extinción (SEMARNAT, 2001). *=especies de mayor valor como indicadores ecológicos (InVal $\geq 90 \%)$. 